\title{
GOLD severity stratification and risk of hospitalisation for COPD exacerbations
}

\author{
M. Lusuardi1 ${ }^{1}$ C. Lucioni2, F. De Benedetto3, S. Mazzi2, \\ C.M. Sanguinetti ${ }^{4}$, C.F. Donner 5
}

\begin{abstract}
GOLD severity stratification and risk of hospitalisation for COPD exacerbations. M. Lusuardi, C. Lucioni, F. De Benedetto, S. Mazzi, C.M. Sanguinetti, C.F. Donner.

Background and Aim. The Italian Costs for Exacerbations in COPD ("ICE") study, following a pharmacoeconomic assessment of costs due to COPD exacerbations (primary endpoint), aimed also at evaluating (secondary endpoint) which clinical factors, among those considered for cost-analysis, may, at follow up, present a risk of new exacerbations and re-admission to hospital.

Materials and methods. A prospective, multicentre study was carried out on COPD patients admitted to 25 Hospital Centres as a result of an exacerbation from October-December 2002. Following discharge, a 6-month follow-up was performed in each patient via three bi-monthly telephone interviews with a questionnaire administered by an investigator clinician.

Results. 570 patients were eligible for data processing, mean age 70.6 years $( \pm 9.5$ standard deviation, $S D)$, males
\end{abstract}

69.2\%. According to GOLD, severity stratification was as follows: moderate $36.4 \%$; severe $31.3 \%$; very severe $32.3 \%$. 282 patients experienced at least one exacerbation at follow up, $42 \%$ of exacerbations requiring hospitalisation. No significant association was seen between exacerbations and GOLD stage or co-morbidities or treatments except LTOT. Conversely, COPD functional severity influenced hospitalisations very significantly, with relative risks 2.6 (95\% Confidence Interval, CI 1.8-3.8) and 2.0 (CI 1.3-2.8) (GOLD very severe versus moderate and severe, respectively), and 1.3 (CI 0.85-2.1) (GOLD severe versus moderate). Hospitalisations were also significantly associated with treatments denoting more severe conditions (oral corticosteroids, oral theophylline, and LTOT).

Conclusions. Severity stratification of COPD patients according to respiratory function classes as outlined in GOLD guidelines and need for LTOT are confirmed as important predictors of hospitalisation for an exacerbation.

Monaldi Arch Chest Dis 2008; 69: 4, 164-169.

Keywords: Chronic obstructive pulmonary disease, Severity stratification, Risk factors, Exacerbations, Hospitalisation.

${ }^{1}$ Department of Pulmonary Rehabilitation, S. Sebastiano Hospital, AUSL RE, Correggio (RE);

2 Wolters Kluwer Health/ADIS International, Milano;

3 U.O.C. di Pneumologia - P.O. "S.S. Annunziata" - ASL Chieti, Chieti Scalo (CH);

4 U.O.C di Pneumologia, A.C.O San Filippo Neri, Roma;

5 Mondo Medico, Multidisciplinary and Rehabilitation Outpatient Clinic, Borgomanero (NO), Italy.

Correspondence: Dr. Mirco Lusuardi, Pulmonary Rehabilitation, S. Sebastiano Hospital, Reggio Emilia AUSL, Via Mandriolo 11, I-42015 Correggio (RE), Italy; e-mail: lusuardimi@ausl.re.it

\section{Introduction}

Chronic obstructive pulmonary disease (COPD) is a leading cause of morbidity and mortality carrying a heavy economic burden worldwide.

Hospitalisations for an exacerbation are the major cost factor for COPD [1] and are per se a risk factor for further hospitalisations [2-4]. The prospective ICE study's primary aim was to evaluate the direct and indirect annual costs due to COPD in a cohort of Italian patients enrolled after hospital admission for an exacerbation. Data demonstrated that hospitalisations accounted for about $40 \%$ of costs, followed by long-term oxygen therapy $(30 \%)$ and drug treatments $(18 \%)[5,6]$.

A further aim of the ICE study was to evaluate which clinical factors among those considered for cost-analysis might influence at follow up the risk of a new exacerbation and re-admission to hospital.

\section{Methods}

A number of hospital pulmonary departments throughout Italy were contacted. In the period October to December 2002, each centre had to enrol the first 30 consecutive patients aged $>40$ years who were admitted to hospital for a COPD exacerbation, independently of whether or not they had had previous hospitalisation for COPD. A COPD exacerbation was defined as an increase in dyspnea, sputum production or sputum purulence.

Only patients with a spirometric confirmation of COPD diagnosis $\left(\mathrm{FEV}_{1} / \mathrm{VC} \leq 0.7\right.$ post-bronchodilator) could be enrolled. The participating centres agreed to adhere to ATS standards for 
spirometry [7]. Only moderate to very severe stages according to GOLD classification [8] were considered for inclusion. Classes of GOLD severity stratification originally considered were moderate/2a, moderate-severe/2b, and severe/3 [5]; to avoid possible confusion for the reader we have opted here-on to use the present classification, since functional characteristics of the respective stages are equivalent: moderate $/ 2$, severe $/ 3$, very severe/4 [8].

Functional severity may vary during the course of an exacerbation, therefore spirometric data obtained at discharge were considered for this study. Milder severity stages were not taken into account because, in general, they do not meet the appropriate criteria for hospital admission. Data registered during the hospital stay pertinent to this study were demographic data, COPD severity and individual co-morbidities. Data were collected by chart reviews from hospital records obtained at admission. According to the frequency of the different comorbidities four main groups were considered for analysis, i.e. cardiovascular, diabetes, other and no co-morbidities [5].

After discharge patients were observed for a 6month follow up in the period January to June 2003 with three telephone interviews every 2 months. The interviewer (the same physician(s) in charge of the study at each centre) registered the new episode(s) of COPD exacerbation and the number of hospitalisations related to an exacerbation, along with treatments, in particular systemic corticosteroids, antibiotics, inhaled corticosteroids, inhaled corticosteroids + long-acting beta2 -agonists in combination, theophylline and longterm oxygen therapy.

The ethical considerations were managed by each local review board. The written consent of each patient was formally requested and obtained for the treatment of personal data for research.

\section{Statistics}

Categorical variables are presented as proportions. Continuous variables are presented as mean and SD or median and interquartile range according to normal or nonnormal type of distibution respectively.

The relative risk $(95 \%$ Confidence Interval CI), of a new exacerbation and re-admission to hospital was calculated for the global population and for the sub-populations stratified according to GOLD severity stages.

The independence between categorical variables was tested with Pearson's chi-square. The difference between parameters was tested with Student's " $t$ " test. The level of statistical significance was set at a $p$ value $<0.05$.

\section{Results}

25 centres across Italy participated in the study. 635 patients were enrolled. However, it was only possible to complete the follow up with three interviews for 570 patients, $30.8 \%$ females, mean age $70.6( \pm 9.5 \mathrm{SD})$. Among patients lost to follow up 43 had died, accounting for a $6.8 \%$ mortality at 6 months. The following data refers only to those 570 patients completing the survey.

\section{Exacerbations}

The number of patients who experienced at least one new exacerbation during follow up was $282(49 \%)$. A certain degree of relationship between the number of exacerbations and the GOLD severity stage is apparent, although not significant at the conventional level $(p=0.07)$ (figure 1$)$.

The number of patients experiencing new exacerbations during the follow up stratified according

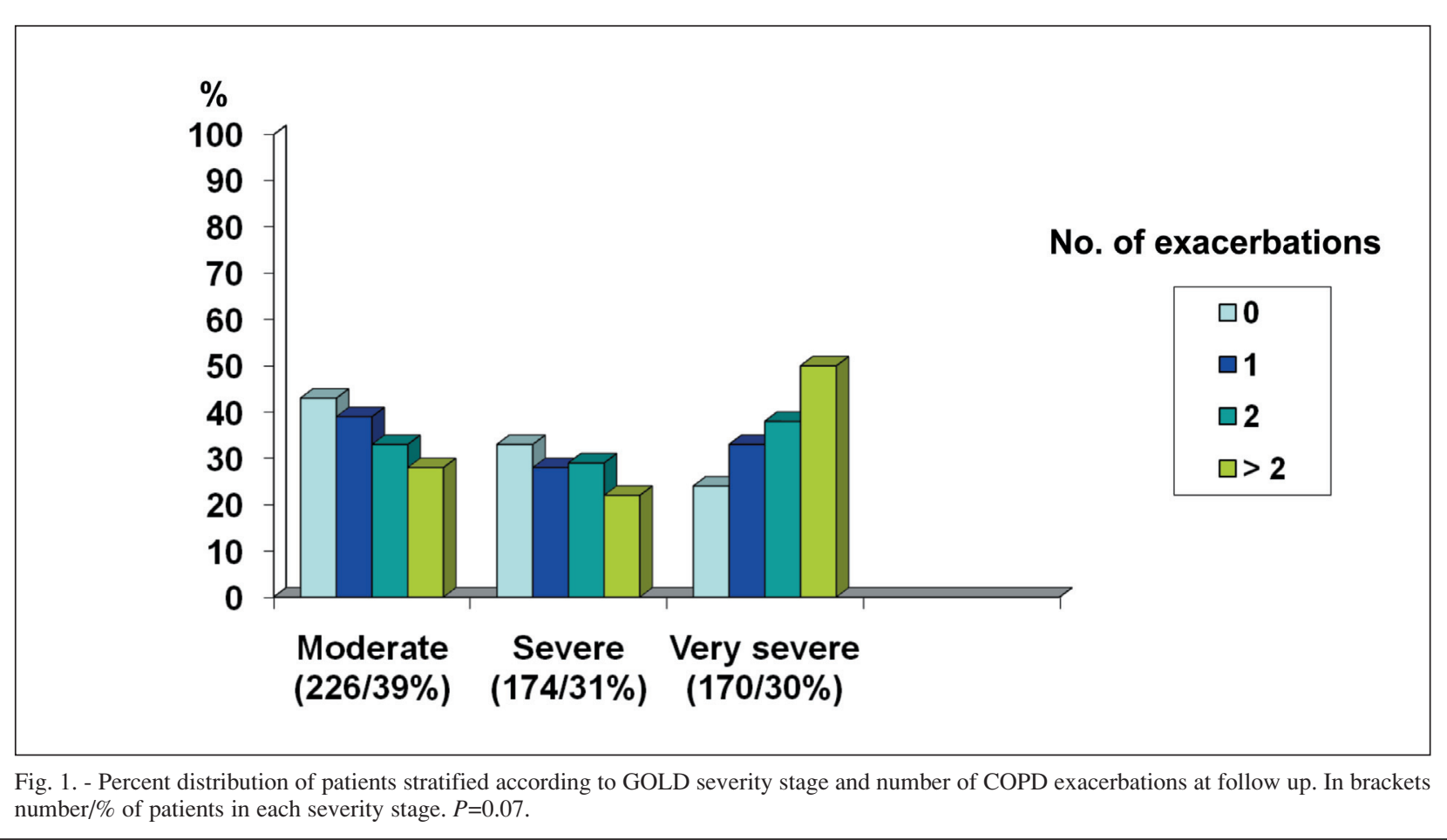


to co-morbidities is shown in table $1.34 .2 \%$ of patients had no co-morbidities, while cardiovascular disorders were the most represented (53.5\%). Again, no significant association was seen between exacerbations and co-morbidities, although the proportion of patients with cardiovascular disease was higher among patients with $>2$ exacerbations.

The risk of an exacerbation was calculated in relation to different treatments. No significant association was found for regular oral corticosteroids (risk of an exacerbation $54 \%$ for the treatment group, 52 cases, versus 49\%, 518 cases, $\mathrm{p}=0.49$ ), inhaled corticosteroids + long-acting $\beta 2$-agonists (risk for an exacerbation $54 \%$ for the treatment group, 159 cases, versus 48\%, 411 cases taking other treatments without corticosteroids, $p=0.2$ ), oral theophylline (risk for an exacerbation $57 \%$ for the treatment group, 98 cases, versus 48\%, 472 cases, $p=0.11$ ), or antibiotic courses (risk of an exacerbation $44 \%$ for the treatment group, 50 cases, versus $50 \%, 520$ cases, $p=0.42$ ). The same evaluation conducted taking into account the different GOLD severity stages, once again, did not show any significant difference (data not shown).

On the contrary, the presence of long-term oxygen therapy was associated with a significant risk of exacerbation ( $59 \%$ for the treatment group, 227 cases, versus $43 \%, 343$ cases, $p=0.0002$ ); the three severity stages contributed in a similar way (59 vs $43 \%$ for moderate, 54 vs $42 \%$ for severe, and 62 vs $47 \%$ for very severe).

\section{Hospitalisations}

Among those 282 patients with at least one episode of COPD exacerbation at the six month follow up 124 (44\%) had to be admitted to hospital.

The likelihood of hospitalisation following an episode of COPD exacerbation was associated in a highly significant way with disease severity (figure 2).

Table 1. - Number (\%) of patients stratified according to the main co-morbidity and number of COPD exacerbations at follow up

\begin{tabular}{lccccc}
\hline & $\begin{array}{c}\text { Cardiovascular } \\
\text { comorbidities }\end{array}$ & Diabetes & $\begin{array}{c}\text { Other } \\
\text { comorbidities }\end{array}$ & $\begin{array}{c}\text { No } \\
\text { comorbidities }\end{array}$ & $\begin{array}{c}\text { Total } \\
(\mathbf{1 0 0 \%})\end{array}$ \\
\hline No of exacerbations & $150(52 \%)$ & $11(4 \%)$ & $18(6 \%)$ & $109(38 \%)$ & 288 \\
\hline 1 & $102(55 \%)$ & $16(9 \%)$ & $10(5 \%)$ & $58(31 \%)$ & 186 \\
\hline 2 & $41(52.5 \%)$ & $7(9 \%)$ & $7(9 \%)$ & $23(29.5 \%)$ & 78 \\
\hline$>2$ & $12(66.7 \%)$ & $1(5.5 \%)$ & 0 & $5(27.8 \%)$ & 18 \\
\hline$p=0.29$ & $305(54 \%)$ & $35(6 \%)$ & $35(6 \%)$ & $195(34 \%)$ & 570 \\
\hline
\end{tabular}

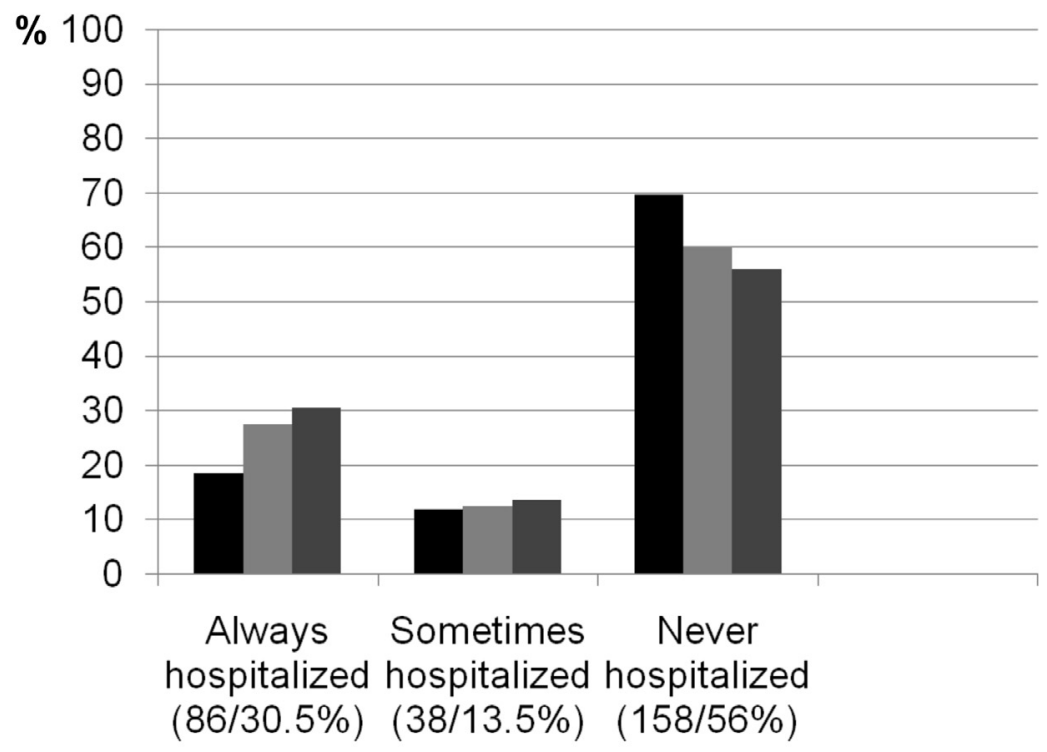

Moderate (102)

Severe (80)

- Very severe (100)

Fig. 2. - Percent distribution of patients stratified according to the GOLD severity stage (no.) with at least one exacerbation at follow up divided according to need for hospitalisation (no. and \%). $P=0.0003$ for likelihood of hospitalisation in relation to disease severity. 
Co-morbidities did not seem to have a significant impact on hospitalisations $(p=0.5)$. The mean length of stay (LOS), 11.7 days (median 10 days), was not influenced by the GOLD severity stage $(p=0.35)$ or the presence of co-morbidities $(\mathrm{p}=0.74)$. For a more sensitive evaluation the $1 \mathrm{st}$ quartile ( $<=8$ days) and the 4th quartile ( $>15$ days) of the LOS were compared but again no significant association was found with the severity stage.

Comparing patients with at least one hospitalisation for a COPD exacerbation with patients who were not hospitalized during the follow up, the risk of hospital admission was significantly linked to the GOLD severity stage $(p<0.0001)$ as shown in table 2, which reports also the respective relative risks comparing GOLD stages very severe versus moderate and severe (2.6 and 2.0 respectively) and GOLD severe versus moderate (1.3).

As for the risk of hospital admission due to a COPD exacerbation, patients on regular therapy with oral corticosteroids had a significantly higher risk of hospitalisation (42\% versus $20 \%$, $p=0.0003$ ); the same was true for regular treatment with oral theophylline (39\% versus 18\%, $p=0.00001)$ and for long-term oxygen therapy ( $32 \%$ versus $15 \%, p=0.00001$ ). On the contrary, regular treatment with inhaled corticosteroids + long-acting $\beta 2$-agonists versus other treatments without inhaled corticosteroids did not influence the probability of hospitalisation (25 versus $20 \%$, $p=0.19$ ).

\section{Discussion}

The ICE study enrolled patients with a severity stage from moderate to very severe according to GOLD [8]. If we refer to a large epidemiological study in USA, these patients, account for a prevalence of about $7 \%$ of the COPD population (which, in turn, constitutes about $6.8 \%$ of the general population - NHANES III) [9]. As a consequence, about $0.5 \%$ of the population in western countries are affected by moderate to very severe COPD.

In the ICE study these patients experienced on average $1.4 \pm 0.07(\mathrm{SD})$ exacerbations per year (excluding the index hospitalisation at recruitment) [5]. At follow up, 44\% of the patients with an exacerbation required rehospitalisation. The average length of stay (LOS) of 11.7 days (median 10 days) was longer in Italian hospitals than in the UK (mean 8.7, median 6 days) [10] or USA (median 9 days) [11]. Price et al. demonstrated a relationship between organisational scores and LOS [10]. Whether there is a relationship between longer LOS and major outcomes would probably merit further investigation.

A six month mortality of $6.8 \%$ compares favourably with different prospective studies in the international literature, that reports higher percentages from 13.4 [12] to 24 [12] to $33 \%$ [11]. These discrepancies are certainly attributable in part to the different average severity of patients, but there may be differences also in the organization of health services deserving further investigation (e.g. longer length of stay, different patterns of antibiotic coverage etc.).

In our analysis of the data, we endeavoured to identify which of the factors considered for the pharmaeconomic assessment analysis (severity stage, co-morbidities and treatments) might have influenced the recurrence of COPD exacerbations, the risk of hospitalisation and the LOS.

Severity stage: a tenuous relationship between severity stage and incidence of exacerbations was found, although not strictly significant. On the contrary, the risk of hospitalisation for a COPD exacerbation was related to the severity stage according to GOLD [8] in a highly significant way.

GOLD guidelines base severity stages mainly on functional criteria (severity of spirometric ab-

Table 2. - Risk of hospitalisation for an exacerbation according to GOLD severity stage

\begin{tabular}{llllll}
\hline & $\begin{array}{l}\text { GOLD stage } \\
\rightarrow \text { no. of patients }(\%)\end{array}$ & Moderate & Severe & Very severe & Total \\
\hline Hospitalisation YES & & $31(14 \%)$ & $32(18 \%)$ & $61(36 \%)$ & $124(22 \%)$ \\
\hline Hospitalisation NO & & $195(86 \%)$ & $142(82 \%)$ & $109(64 \%)$ & $446(78 \%)$ \\
\hline Total (100\%) & 226 & 174 & 170 & 570 \\
\hline Risk of hospitalisation & & $14 \%$ & $18 \%$ & $36 \%$ & $22 \%$ \\
\hline Relative risk & $\begin{array}{l}\text { very severe } \\
\text { vs moderate }\end{array}$ & & & & 2.6 \\
\cline { 2 - 5 } & $\begin{array}{l}\text { very severe } \\
\text { vs severe }\end{array}$ & & & & 2.0 \\
\cline { 2 - 5 } & $\begin{array}{l}\text { severe } \\
\text { vs moderate }\end{array}$ & & & & $1.8-3.8)$ \\
\hline
\end{tabular}


normality), but also on the presence of complications such as chronic respiratory failure [8], a point equivalent to need for LTOT in the patients enrolled in our series. The level of symptoms as reported in the GOLD guidelines does not seem to have a real discrimination role, unless objective measurement of cough, dyspnea and expectoration are consistently used prospectively. Recent literature has demonstrated that the best outcome predictors are multifactorial scores including functional data only as a partial component such as in BODE (Body mass index, airways Obstruction, Dyspnea, Exercise performance). Actually, the BODE significantly predicts the risk of hospitalisation [14]. According to our data, simple functional stratification with spirometry remains a strong predictor of hospitalisation, which is per se a major critical event in the natural history of COPD and a further strong predictor of survival, re-exacerbation, and re-hospitalisation [2-4].

The LOS was not influenced by the severity stage, the most likely explanation being that a more complex combination of clinical and organisational factors can best predict the LOS, aside from the basic disorder [10].

Comorbidities: despite their significant role in COPD mortality [15], in our series comorbidities did not seem to influence the recurrence of exacerbations or the risk of hospitalisation. An eventual methodological limitation is our evaluation of individual co-mordidities rather than a weighted comorbidity score such as the Charlson Index [16].

Treatments: although the study was not designed to assess the influence of individual drug classes on exacerbations and hospitalisations [17], it is interesting to note that none of the drug therapies (both regular or for the exacerbation) seemed to influence the risk of exacerbation recurrence, with the sole exception of long-term oxygen therapy (LTOT). This is probably because LTOT is a strong indicator of severity, while the other treatments do not correlate with the presence of chronic respiratory failure. For the same reason, LTOT again strongly denoted a risk of hospitalisation, but in this case also oral corticosteroids and oral theophylline used at follow up were significantly associated with hospitalisations. This is not in contrast with a recent systematic review and meta-analysis demonstrating that systemic corticosteroids are effective in reducing treatment failures [18], as our data refers to regular use of corticosteroids, a finding supported by other authors [19].

The strengths of our study are its prospective design with quite a large multicentre cohort of moderate to very severe patients admitted to hospital for an exacerbation, unselected from the point of view of concurrent disorders and medications.

Among the weaknesses, we must underline a short follow up of only six months and the fact that the patients were representative of a limited part of the COPD population, albeit the most problematic from a clinical and economic point of view [5].

COPD patients may largely benefit from drug treatments or rehabilitation (8) or integrated care programmes [20], but the appropriate choice of management options cannot be based solely on clinical grounds. Severity stratification is mandatory as all the evidence in the literature is related to selected classes of severity. Recent reports have highlighted the importance of different severity measures apart from spirometry, such as dyspnea, functional performance [21], quality of life, BMI, particularly combined in composite scores such as the BODE, or comorbidities or history of frequent exacerbations/hospitalisations etc. [12-15]. Unfortunately, current diagnostic standards are far lower than expected since a large proportion of COPD diagnoses (even in hospital) is not supported by spirometry and bronchodilator test $[22,23]$; therefore management remains largely empirical and outcome evaluation often inconsistent.

In conclusion, our data confirms the importance of the often neglected spirometry stratification of COPD severity as a hard determinant of hospitalisation as a major outcome. Recent evidence obtained in a large series of COPD patients similar to ours, but selected for a pharmacologic trial, reached the same conclusion, i.e. that spirometry is strongly predictive of hospitalisation for an exacerbation [19]. A further point is that not only functional stage, but also regular therapies typically linked to severity or complications (oral corticosteroids, LTOT, theophylline as a second line drug) should be taken into account when estimating the prognosis of COPD patients. On the contrary, our results on co-morbidities versus the risk of re-exacerbation/hospitalisation were inconclusive, due to methodological limitations.

It is imperative to improve standards of care for patients with suspected COPD in order to stratify severity and predict risk of re-exacerbation and hospitalisation so as to ensure an appropriate management of cases at higher risk.

Acknowledgments. The ICE study is a project of AIMAR (Associazione Scientifica Interdisciplinare per lo Studio delle Malattie Respiratorie), a scientific association for the multidisciplinary study of respiratory disorders. The study was supported by an unrestricted grant by Pfizer-Italy, Roma, and Boehringer-Ingelheim Milan, Italy. We would like to thank the following Italian Pulmonary Medicine Specialists and Departments that participated in the study: Az. Osp. San Luigi, Orbassano (TO) - Spedali Civili, Brescia - Az. Osp. della Provincia di Lodi, Lodi - A.O. San Carlo Borromeo, Milano - Ospedale L. Sacco, Milano - P.O. S. Marta "Ospedale Maggiore di Crema", Crema - Ospedale Santa Maria degli Angeli, Pordenone - Ospedale Civile di Treviso, ULSS n. 9, Treviso - Ospedale Civile di Piacenza, Piacenza - Arcispedale "S. Anna", Ferrara - Ospedale di Cisanello, Pisa - Ospedale Mazzoni, ASL 13, Ascoli Piceno - Ospedale Regina Apostolorum, Albano Laziale (Roma) A.C.O. S. Filippo Neri, Roma - P.O. S. Camillo De Lellis, Chieti - Centro Medico Italo-Australiano, Cava de' Tirreni (SA) - Casa di Cura Clinic Centre S.p.A., Napoli - Az. Osp. OO. RR. S. Giovanni di Dio, Salerno - Ospedale D'Avanzo, Foggia - Ospedale "A. Galateo", ASL LE/1, Lecce - Az. Osp. Pugliese Ciacco, Catanzaro - Ospedale "Mariano Santo", Cosenza - Ospedale Tommaselli Catania - Ospedale R. Binaghi, USL 8, Cagliari - Università degli Studi di Sassari, Sassari. 
care on patient outcome following admission for acute COPD exacerbation. Thorax 2006; 61: 837-842.

11. Connors AF, Dawson NV, Thomas C, et al. Outcomes following acute exacerbation of severe chronic obstructive pulmonary disease. Am J Respir Crit Care Med 1996; 154: 959-967.

12. Almagro P, Calbo E, Ochoa de Echaguen A, et al. Mortality after hospitalisation for COPD. Chest 2002; 121: 1441-1448.

13. Gunen H, Hacievliyagil SS, Kosar F, et al. Factors affecting survival of hospitalised patients with COPD. Eur Respir J 2005; 26: 234-241.

14. Ong K-H, Earnest A, Lu S-J. A multidimensional grading system (BODE Index) as predictor of hospitalisation for COPD. Chest 2005; 128: 3810-3816.

15. Sin DD, Anthonisen NR, Soriano JB, Agusti AG. Mortality in COPD: role of comorbidities. Eur Respir $J$ 2006; 28: 1245-1257.

16. Charlson ME, Pompei P, Ales KL, et al. A new method of classifying prognostic comorbidity in longitudinal studies: development and validation. J Chronic Dis 1987; 40: 373-383.

17. Sanguinetti CM, Corrao G, Ochan Bilama M, et al. An Italian investigation of pathogens causing COPD exacerbation and their resistance to common antibiotics (The EOLO Study). Multidisciplinary Respiratory Medicine 2008; 1: 13-21.

18. Quon BS, Gan WQ, Sin DD. Contemporary management of acute exacerbations of COPD. Chest 2008; 133: 756-766.

19. Niewoehner DE, Lokhnygina Y, Rice K, et al. Risk indexes for exacerbations and hospitalisations due to COPD. Chest 2007; 131: 20-28.

20. Casas A, Troosters T, Garcia-Aymerich J, et al. Integrated care prevents hospitalisations for exacerbations in COPD patients. Eur Respir J 2006; 28: 123-130.

21. Lareau SC. Importance of functional performance assessment for severe COPD. Multidisciplinary Respiratory Medicine 2008; 1: 30-33.

22. Han MLK, Kim MG, Mardon R, et al. Spirometry utilization for COPD. How do we measure up? Chest 2007; 132: 403-409.

23. Lusuardi M, Orlandini D. Underutilization of spirometry for the diagnosis of COPD. Chest 2008; 133; 313-314.

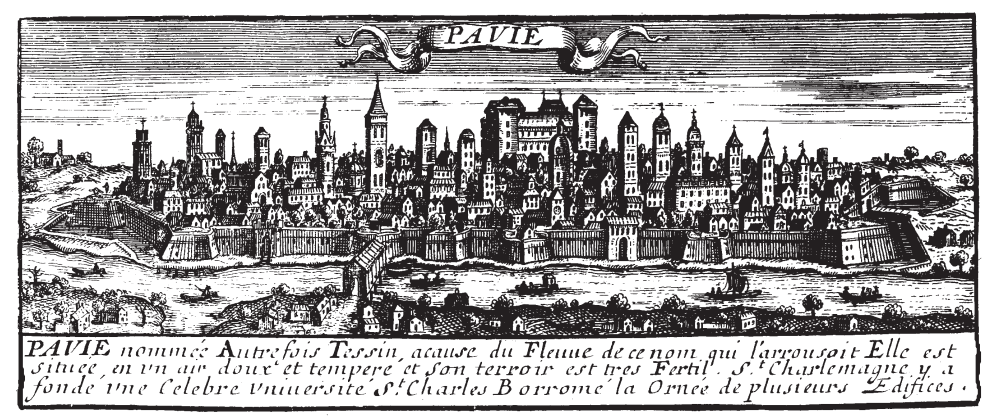

